$16^{\text {th }}$ International Conference on Information Systems and Technology Management - CONTECSI2019

DOI: 10.5748/16CONTECSI/LIS - 6273

\title{
INFORMATION AND KNOWLEDGE MANAGEMENT ON THE DATA WEB
}

\section{GESTÃO DA INFORMAÇÃO E CONHECIMENTO NA WEB DE DADOS}

Francisco Carlos Paletta - Universidade de São Paulo, Brasil - fcpaletta@usp.br José Antonio Moreiro Gonzalez - Universidade Carlos III de Madrid - Espanha jamore@bibuc3.es

Waldomiro de Castro Santos Vergueiro - Universidade de São Paulo - Brasil. wdcsverg@usp.br

Resumo: A colaboração tem por objetivo estabelecer uma proposta de pesquisa e contribuição da Ciência da Informação para o entendimento do processo de acesso, uso e apropriação da informação e do conhecimento na era digital. Com o estudo objetiva-se verificar a partir da apropriação que as Bibliotecas e os Repositórios Digitais têm feito das tecnologias colaborativas em ambientes de informação digital e sistemas abertos, as melhores práticas na gestão da infraestrutura de recursos computacionais com foco em propor orientação metodológica que possa indicar práticas de Governança da Tecnologia da Informação em Bibliotecas Digitais. O texto está estruturado em três grandes apartados: (i) Estudo dos Modelos de Busca e Comportamento Informacional do Usuário da Informação na Web; (ii) Infraestrutura Tecnológica e Recursos Computacionais Aplicados em Bibliotecas Digitas; e (iii) Ferramentas de Busca, Acesso, Apropriação, e Uso da Informação na WEB.

Palavras-chave: Gestão da Informação. Gestão do Conhecimento. Web de Dados. Usuário da Informação. Sociedade em Rede.

Abstract: This proposal aims to establish the working plan of the research for the contribution of the information Science to the process of access, use and appropriation of the information and knowledge in the digital era. The Research Project is structured in three phases: (i) Information Search Models Study and User Information Behavior in the Web; (ii) Technological Infrastructure and Computational Resources applied to Digital Libraries; and (iii) Search Tools, Access, Appropriation and Information Use on the Web. The research has been conducted to verify best practices in infrastructure management of computing resources, considering Libraries and Digital Repositories appropriation of collaborative technologies in digital information environments and open systems. The focus is on methodological orientation proposal that can indicate practices in Information Technology Governance in Digital Libraries.

Keywords: Information Management. Knowledge Management. Web of Data. Information User. Network Society. 


\section{INTRODUÇÃO}

Este estudo tem por objetivo verificar, a partir da apropriação que as Bibliotecas e os Repositórios Digitais têm feito das tecnologias colaborativas em ambientes de informação digital e sistemas abertos, as melhores práticas na gestão da infraestrutura de recursos computacionais com foco em propor orientação metodológica que possa indicar práticas de Governança da Tecnologia da Informação em Bibliotecas Digitais.

Atualmente, há a necessidade de novas propostas para abordagens gerenciais e estratégicas associadas à utilização da Tecnologia da Informação na apropriação e geração de conhecimento no contexto da WEB. Neste trabalho, nossa proposta é identificar e discutir as tendências nesse campo, em especial interesse aquelas que influenciam o contexto da chamada Biblioteca Digital, de modo que, cada vez mais, a tecnologia possa proporcionar a criação e obtenção de valor ao usuário da informação. Espera-se que o desenvolvimento dessa área leve a um maior entendimento dos recursos da tecnologia colaborativa utilizados em ambientes informacionais digitais. A inserção dessas tecnologias consiste em inovação seja em processos ou em serviços que deve estar vinculada à tradição e à missão das bibliotecas e dos repositórios de informação (Harbo and Hansen, 2012).

Toda ciência é uma atividade social determinada por condições históricas e socioeconômicas. Desta forma a sociedade da informação necessita de uma ciência que estude as propriedades da informação e os processos de sua construção, comunicação e uso. Hoje, o objeto da Ciência da Informação não é mais o mesmo da biblioteconomia e de suas veneráveis disciplinas coirmãs. Não é mais a biblioteca e o livro, o centro de documentação e o documento, o museu e o objeto, mas é a informação que apresenta foco (Le Coadic, 1996).

O primeiro princípio da Web, proposto pelo Consórcio W3C Brasil - World Wide Web, afirma que o principal valor da Web é o social. Mais do que tecnológico, este é um ambiente de comunicação humana, de transações comerciais, de oportunidades para compartilhar conhecimentos e, para ser um ambiente universal, deve estar disponível para todas as pessoas, independentemente dos equipamentos e softwares que utilizem, principalmente da cultura em que se inserem, da localização geográfica, das habilidades físicas ou mentais, das condições socioeconômicas ou de instrução. A universalidade da Web só pode ser garantida e aprofundada com um modelo de governança democrático e pluralista que tenha foco no acesso por todos e na sua própria evolução tecnológica (Comitê, 2010).

\section{SOCIEDADE EM REDE E O USUÁRIO DA INFORMAÇÃO}

No novo contexto mundial definido pela globalização e pela mudança tecnológica, o conhecimento tornou-se principal riqueza das nações, das 
empresas e das pessoas, podendo também vir a constituir o principal fator de desigualdade. A Sociedade da Informação é a pedra angular da Sociedade do Conhecimento. O conceito de "Sociedade da Informação" está relacionado à idéia da "inovação tecnológica", enquanto o conceito de "Sociedade do Conhecimento" inclui uma dimensão de transformação social, cultural, econômica, política e institucional, assim como uma perspectiva mais pluralista e de desenvolvimento. O conceito de "sociedade do conhecimento" expressa a complexidade e o dinamismo das mudanças que estão ocorrendo. 0 conhecimento em questão não só é importante para o crescimento econômico, mas também para fortalecer e desenvolver todos os setores da sociedade (Clavero, 2005).

O comportamento de busca, acesso, e uso de informação são modelados pelo estilo cognitivo do indivíduo e por fatores que geram o encontro do usuário com os sistemas de informação ou as consequências de tal confronto. É preciso atentar para o fato de que não é mais possível se limitar à tarefa de localizar fontes de informação, não levando em consideração as tarefas de interpretação, formulação e aprendizagem envolvidas no processo de busca e acesso a informação. A velocidade de acesso à informação no universo digital requer serviços ao usuário que estejam centrados no significado da busca mais do que meramente na localização da fonte (Wilson, 1981).

Considerando as sete faces da Information Literacy: Tecnologia da Informação, Fontes de Informação, Processo de Informação, Controle da Informação, Construção do Conhecimento, Extensão do Conhecimento e Inteligência (Bruce, 2003), podemos afirmar que, as Bibliotecas Digitais e as Unidades de Informação, são vistas como modelo de ambiente informacional e como espaço de aprendizagem. Neste contexto, observa-se o surgimento uma nova forma de interatividade entre usuário e a Internet: a Web Semântica - uma Web Inteligente com toda sua informação organizada de forma que não somente seres humanos possam entendê-la, mas principalmente máquinas inteligência artificial.

A construção de uma Internet mais inteligente caminha na direção de produzir uma revolução no universo digital da organização da informação e do conhecimento. Com o uso de novas tecnologias é imperativo o uso das Tecnologias da Informação e das Comunicações em tornar os procesos de busca de informação e a geração de novo conhecimento mais ágeis. É neste ponto que surge um novo usuário da informação com novas demandas por recursos computacionais e novas capacidades em produzir novos conhecimentos (Castells, 2005).

Literacia informacional e digital tem constituído, nos últimos anos, novo campo de pesquisa que interessa à educação, à Ciência da Informação e às ciências cognitivas. O desafio está em, num primeiro momento, aprender a utilização básica dos recursos tecnológicos - literacia digital - e a seguir apropriar-se dos mesmos para gerar novos conhecimentos - literacia informacional. Entretanto 
esta apresentação reducionista de ambas as formas de literacia gestadas no bojo da sociedade em rede não dá conta de delinear as profundas rupturas imersas nas mesmas. Mais do que criar tecnologias intelectuais inovadoras o verdadeiro desafio do campo da informação seria contribuir para criar, na sociedade em rede, uma consciência da imensa riqueza coletiva, em escala mundial, que o acesso gratuito ao domínio público mundial da informação representa (Freire, 2010).

No contexto do Universo Digital é importante considerar a dificuldade encontrada pelas Bibliotecas e Unidades de Informação com suas coleções caríssimas em concorrer com acervos digitais, bibliotecas virtuais, e os motores de busca, oferecendo informações de forma imediata e a custo zero. Neste contexto, observamos a desconstrução do conceito de autor individual e a emergência dos coletivos digitais; a nova valoração das fontes de informação bem como as novas competências que os profissionais da informação precisam desenvolver para atuar como gestores da informação em ambientes em rede (IFLA, 1997). Uma representação genérica das atividades da biblioteca, vistas pelos olhos de um avaliador, constitui um modo conveniente de se enfocar a avaliação dos serviços bibliotecários. Acredita-se que o objetivo de longo prazo da biblioteca seja produzir certos resultados na comunidade a ser atendida (Lancaster, 2004).

Nesse cenário de transformações reais, cresce a responsabilidade social dos profissionais da informação, tanto como produtores de conhecimento no campo científico quanto como facilitadores na comunicação da informação para usuários que dela necessitem, na sociedade, independentemente dos espaços sociais onde vivem e dos papéis que desempenham no sistema produtivo (Freire, 2010).

\section{INFORMAÇÃO E CONHECIMENTO NA WEB DE DADOS}

Uma vez que os saberes científicos e as inovações tecnológicas estão desigualmente repartidos entre países ricos e pobres, por níveis educacionais e faixas etárias, a problemática da diversidade cultural e os estudos sobre ela devem fazer parte da consideração teórica, da investigação empírica e do planejamento de políticas neste campo. Também é necessário delimitar o alcance da posição oposta, que afirma, a partir da antropologia, que todas as sociedades, em todas as épocas, foram sociedades do conhecimento, ou seja, que todo o grupo humano dispôs de um conjunto de saberes apropriado ao seu contexto e aos seus desafios históricos (Choo, 2003).

A organização do conhecimento liga os três processos de uso estratégico da informação - a criação de significado, a construção do conhecimento e a tomada de decisões - num ciclo contínuo de aprendizagem e adaptação que podemos chamar de ciclo do conhecimento. Entre os elementos mais importantes que 
influenciam o uso da informação estão às atitudes do indivíduo em relação à informação e a sua busca, atitudes essas que são fruto da educação, do treinamento, da experiência passada, das preferências pessoais. O risco aqui é de uma super simplificação, de ver o usuário da informação como alguém que quer extrair informações específicas e definitivas no menor tempo possível, ou como alguém disposto a investir esforço para buscar e explorar bases de dados. A verdade é que os indivíduos oscilam continuamente entre extrair e explorar, e que o uso da informação é um processo confuso, desordenado, sujeito aos caprichos da natureza humana, como qualquer outra atividade (Canclini, 2009).

A Internet é provavelmente a mais sofisticada tecnologia de informação e comunicação atualmente disponível para a sociedade, em função da sua forma de organização e de seus impactos nas esferas tecnológica, social, econômica e política. Ela é também a infraestrutura necessária para uma de suas maiores e mais conhecida aplicação: a Web - responsável pela popularização da Internet, a ponto de hoje ser confundida com esta. Internet e Web são, portanto, conceitos distintos. A Web pode ser definida, como a parte da Internet acessada por meio de navegadores, ou browsers. O impacto do uso da Internet e da Web na sociedade, nos indivíduos e nas organizações tornou-se objeto de pesquisa, extrapolando o campo especializado da computação aplicada, e atingindo áreas de estudos organizacionais e sociológicos (Russel, 2013). Por ser essencialmente dinâmica e sem fronteiras, tanto do ponto de vista físico como virtual, é importante que seja conhecida em detalhes, tanto para assegurar sua livre transformação quanto para permitir sua disponibilidade, confiabilidade e acessibilidade por todos.

A Web é uma rede com conteúdos interligados através de documentos de hipertexto. Seu mapeamento é possível por processos de análise e coleta sucessiva de páginas de conteúdo a partir de um conjunto de localizações de documentos previamente conhecido. Tais buscas são feitas de forma automática por programas de computador normalmente denominados crawlers, coletores ou batedores. Mesmo neste mapeamento automático, o conjunto inicialmente assumido de localizações a partir do qual a pesquisa é feita influencia o resultado obtido. Além disso, nem toda a Web está interligada: existem muitos subconjuntos de documentos interligados entre si, de tamanhos variados, sem ligação com o restante da rede, ou seja, formando ilhas de informação. Neste contexto, a Ciência da Informação tem muito em contribuir para com o melhor entendimento e condições de uso potencial dos recursos da Web de Dados.

As constantes mudanças nas formas de organização tradicional da informação presentes em ambientes informacionais digitais são reflexos da incorporação das Tecnologias de Informação e Comunicação (TICs), como no caso das bibliotecas e repositórios digitais, que armazenam, preservam, disseminam e permite o acesso a produção intelectual da comunidade científica e acadêmica, visando contribuir para o aumento da visibilidade e do valor da instituição ao agregar recursos que possibilitam o processo de construção do conhecimento, a partir da participação colaborativa aplicada em diferentes ambientes (Witten and 
Bainbridge, 2009).

A Web Semântica ou Web de Dados é a terceira geração da Internet. Esta nova geração prevê que os conteúdos online estarão organizados de forma Semântica, muito mais personalizada para cada usuário, sites e aplicações inteligentes, e publicidade baseada nas pesquisas e nos comportamentos de busca, acesso e apropriação na informação no universo digital. Esta nova Web também pode ser chamada de "Web Inteligente".

A Web Semântica segue uma filosofia com princípios de leitura e escrita de natureza participativa, em que cada usuário pode intervir diretamente na escolha e introdução de dados no âmbito de cada site. É cooperativa, uma vez que compartilha idéias, preferências, informações e conhecimento. É interativa, na medida em que, através de toda a gama de recursos multimídia, é possível um diálogo simultâneo com os usuários. É democrática, pois sob essa filosofia existe liberdade de expressão, de pensamento, e, sobretudo, de trânsito de informações, independentemente dos interesses de cada um. É também sociotécnica, pois, através de todas as suas características, é possível um intercâmbio de culturas, religiões, etnias e outros.

No contexto da Biblioteca 2.0 é possível afirmar que muito do que foi produzido como inovação nos serviços oferecidos pelas Bibliotecas na primeira revolução da Web estava associada a serviços estáticos. Por exemplo, catálogos online de acesso público (OPAC) exigem que os usuários busquem a informação, embora muitos estejam iniciando a incorporar técnicas da Web 2.0 relativas à pesquisa de dados. Do mesmo modo, a primeira geração de Biblioteca online foi elaborada através de textos tutoriais estáticos e que não respondiam às necessidades dos usuários, nem permitiam que interagissem uns com os outros. As Bibliotecas, porém, tem evoluído para uma estrutura mais interativa, fazendo uso de meios de comunicação social rico em tutoriais, e com o uso de banco de dados mais sofisticados.

A Web 2.0 nas Bibliotecas pode ser uma ferramenta que possibilite a gênese de uma base de conhecimento a partir da inteligência coletiva, como também ferramenta para a gestão do conhecimento que facilite, de maneira interativa, a descoberta dos mesmos. Passamos de uma Biblioteca para o usuário para uma Biblioteca com o usuário. Neste integrar e complementar estes dois paradigmas, devemos também considerar, conhecer, explorar e avaliar as novas ferramentas de comunicação, organização, participaçao e construção coletiva do conhecimento que estão disponíveis na Web. São aplicações, na sua maioria intuitiva, gratuita e que respondem as novas necessidades de informação e participação da comunidade de usuários. Deste modo, fica clara a transformação, ou seja, a nova abordagem da relação entre informação e conhecimento no contexto das bibliotecas e dos repositórios digitais, ao contrário dos ambientes tradicionais que só permitia ao usuário uma única forma de se relacionar com o conteúdo armazenado fisicamente (Kuhlthau, 2004). 
De acordo com o Observatorio para a Cibersociedad (2009), atualmente, não só o usuário participa como também adiciona conteúdos que ao serem compartilhados em outros recursos colaborativos passam a receber comentários dos membros vinculados a sua comunidade, agregando valor à informação compartilhada, além de criar vários caminhos para a localização do próprio recurso. Assim, temos uma nova relação e interação na qual aumenta o nível sofisticado de atitudes sociais eletrônicas.

A Web 1.0 conecta informação. O usuário desempenha o papel de espectador, o conteúdo é pouco interativo. A Web 2.0 conecta pessoas. O foco está na construção coletiva do conhecimento. A essência da Web Social é permitir que os usuários não sejam mais apenas espectadores, e sim que eles se tornem colaboradres. A Web Semântica ou Web de Dados conecta conhecimento. Os algoritmos serão capazes de interpretar nossas preferências e guiar nossa navegação pela Web. Está ligada a um conjunto de tecnologias mais eficientes para ajudar os computadores a organizar e analisar a informação disponível na rede. Fará com que os aplicativos baseados na Web sejam Open-Source e viabilizar a interatividade em diversas áreas da Web.

O universo da informação digital se expande no contexto do que hoje chamamos de Big Data onde a busca por informações fica cada vez mais difícil uma vez que o universo digital esta constituido por dados não estruturados que precisam ser organizados, acessados, apropriados e então utilizados na produção de novos conhecimentos. A Web de Dados virá com o objetivo de organizar estas informações para que os usuários tenham mais facilidade na busca da informação. A busca Semântica como é chamada os buscadores da Web de Dados, organizam informações por assuntos determinados, conectando conhecimentos. A busca Semântica da Web de Dados dividirá os resultados em categorias para que a busca da informação seja mais rápida e organizada.

A Figura 1 relaciona o Grau de Conectividade da Informação com o Grau de Conectividade Social de acordo com a evolução tecnológica: Web - Conectando Informação, Web Social - Conectando Pessoas, Web Semântica - Conectando Conhecimento e a Metaweb - Conectando Inteligência. 


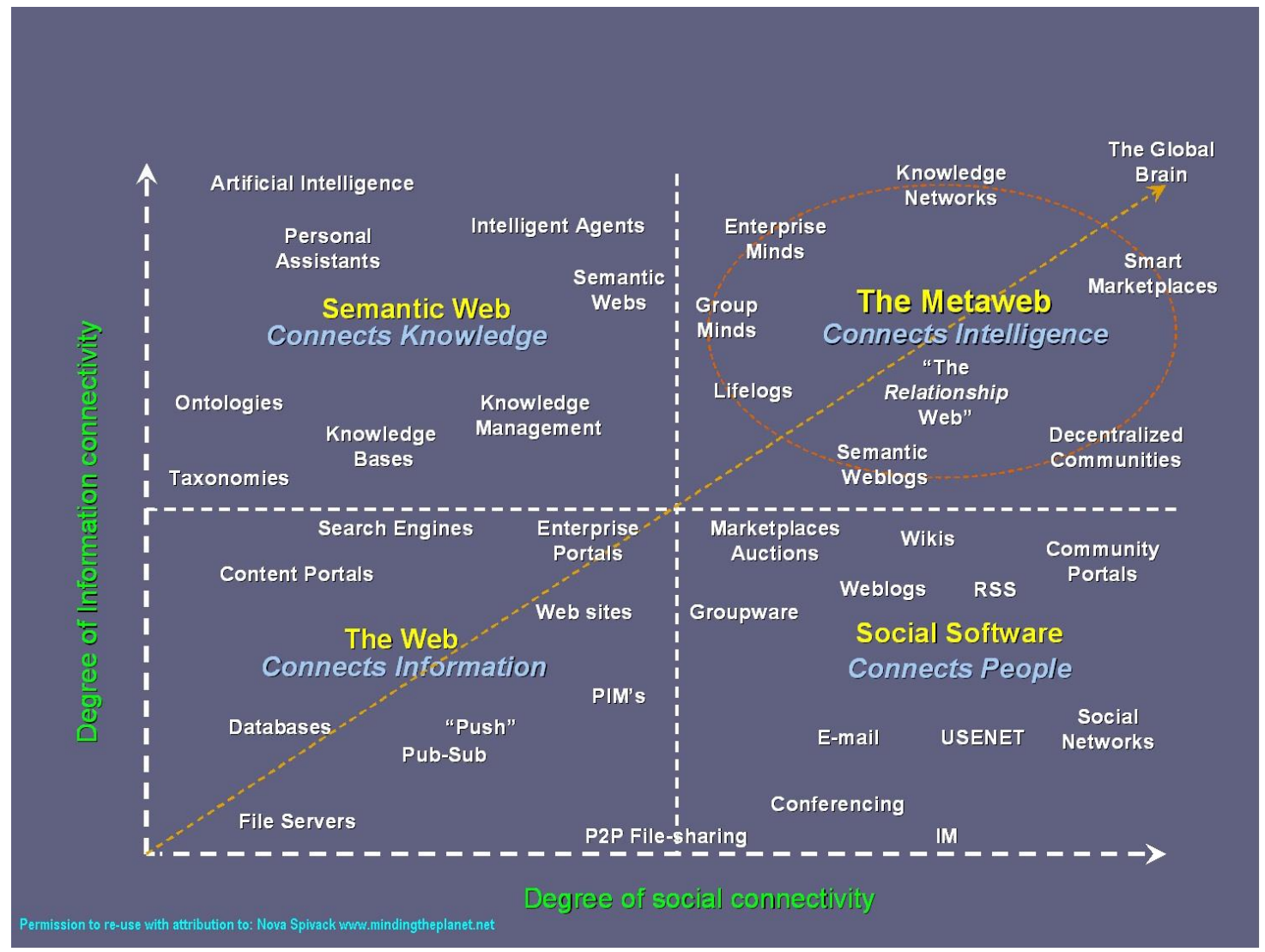

Figura 1: Evolução da WEB

Fonte: http://www.novaspivack.com/science/new-version-of-my-metaweb-graph-the-future-ofthe-net

Biblioteca 3.0 refere-se a Bibliotecas utilizando recursos como a Web Semântica, a computação em nuvem, dispositivos móveis, e uso de tecnologias da informação, para facilitar a disseminação do conteúdo gerado pelo usuário e a atuação em redes de colaboração. O resultado final da Biblioteca 3.0 é a expansão da "Biblioteca sem fronteiras", onde as coleções podem ser disponíveis aos usuários da Biblioteca, independentemente da sua localização física. Biblioteca 3.0 é um complemento virtual para os espaços físicos da Biblioteca (Belling, 2010).

A inovação tecnológica constitui uma ferramenta essencial para aumentar a produtividade e a competitividade das organizações, assim como para impulsionar o desenvolvimento econômico da sociedade. O desenvolvimento não deriva de um mero crescimento das atividades econômicas existentes, mas reside fundamentalmente em um processo qualitativo de transformação da estrutura produtiva no sentido de incorporar novos produtos e processos e agregar valor à produção por meio da intensificação do uso da informação e do conhecimento (OECD, 2005). 
A Universidade atua como organismo gerador, transmissor e receptor de conhecimentos e a Biblioteca através dos Serviços de Informação, torna-se consciente de sua função intermediadora realizando os processos documentários e preservando a informação para sua próxima transformação em conhecimento em uma espiral de evolução científica e tecnológica. Neste contexto a Universidade tem como foco amplificar a produção de tecnologia, inovação, processos, reflexão, política pública e formação de profissionais de qualidade e a Biblioteca atua como agente de socialização de conhecimento e novos saberes. As funções básicas da Biblioteca derivam dessa dinâmica social que, em um movimento circular, fornece insumos para sua própria continuidade. Dentro dessa dinâmica, visualizamos as funções de:

- Armazenagem do Conhecimento: desenvolvimento de coleções, memória da produção científica e tecnológica, preservação e conservação;

- Organização do Conhecimento: qualidade de tratamento temático e descritivo que favoreça o intercâmbio de registros entre Bibliotecas e sua recuperação;

- Acesso ao Conhecimento: a exigência de informação transcende o valor, o lugar e a forma e necessita de acesso. Por isso devemos pensar não só em fornecer a informação, mas possibilitar o acesso simultâneo de todos.

Essas três funções estão presentes em toda a evolução do processo de socialização

do conhecimento realizado pela Universidade ao longo do tempo, mesmo considerando

permanente mudança dos formatos documentários para registro do conhecimento e seu modo de acesso. A Biblioteca insere-se neste contexto cujos objetivos maiores são o desenvolvimento educacional, social, político e econômico da sociedade humana (Fujita, 2005).

\section{A ERA DIGITAL}

A Ciência da Informação no contexto da era digital deve ter em sua agenda a necessidade de novas propostas para abordagens gerenciais e estratégicas associadas à utilização da Tecnologia da Informação na apropriação e geração de conhecimento no contexto da Web de Dados. Um importante desafio está em identificar e discutir as tendências nesse campo, em especial interesse aquelas que influenciam o contexto da chamada Biblioteca Digital, de modo que, cada vez mais, a tecnologia possa proporcionar a criação e obtenção de valor ao usuário da informação. Espera-se que o desenvolvimento dessa área leve a um maior entendimento dos recursos da tecnologia colaborativa 
utilizados em ambientes informacionais digitais. A inserção dessas tecnologias consiste em inovação seja em processos ou em serviços que deve estar vinculada à tradição e à missão das Bibliotecas e dos repositórios de informação digital.

Estrategicamente uma área de pesquisa relevante para a Ciência da Informação está em estudar a apropriação que as Bibliotecas e os Repositórios Digitais têm feito das tecnologias colaborativas em ambientes de informação digital e sistemas abertos bem como a gestão da infraestrutura de seus recursos computacionais na configuração tecnológica dos Serviços de Informação orientados ao usuário da informação na Web.

Os critérios de escolha e a estratégia de coleta e análise dos dados convergem para o instrumento de pesquisa, que pode ser desenvolvida a partir do equacionamento das dimensões e fronteiras investigadas na pesquisa de campo. A Figura 2 apresenta as dimensões consideradas na proposta de estudo:

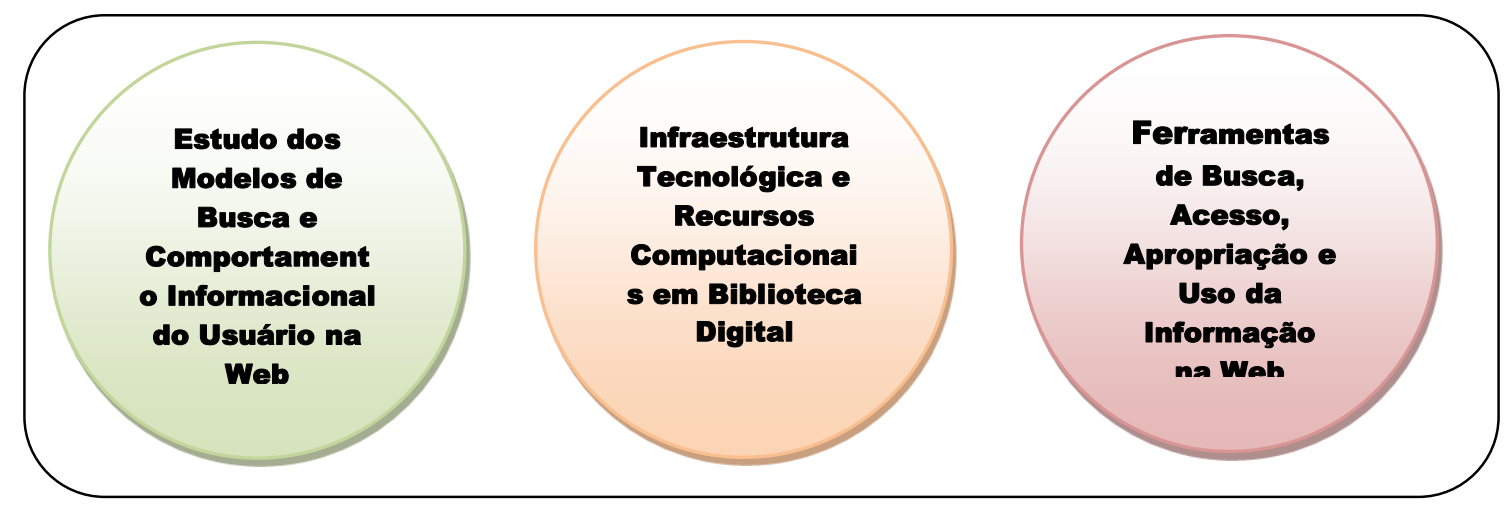

Figura 2: Dimensões Consideradas na proposta de estudo

Fonte: Autor

Vale destacar que se trata de um estudo que tem por objetivo gerar conhecimentos para aplicação prática e solução de problemas definidos. Quanto a abordagem, caracteriza-se como um tipo de pesquisa qualitativa para melhor compreender e classificar os processos relativos ao tema em estudo.

A proposta de estudo está estruturada em três fases: (i) Estudo dos Modelos de Busca e Comportamento Informacional do Usuário da Informação na Web; (ii) Infraestrutura Tecnológica e Recursos Computacionais Aplicados em Bibliotecas Digitas; e (iii) Ferramentas de Busca, Acesso, Apropriação, e Uso da Informação na WEB. Com o estudo objetiva-se verificar a partir da apropriação que as Bibliotecas e os Repositórios Digitais têm feito das tecnologias colaborativas em ambientes de informação digital e sistemas abertos, as melhores práticas na gestão da infraestrutura de recursos computacionais com foco em propor orientação metodológica que possa indicar 
práticas de Governança da Tecnologia da Informação em Bibliotecas Digitais.

O estudo tem por escopo confrontar a utilização dos modelos de estudo de usuários da informação conhecidos com os resultados de pesquisas científicas para buscar documentar e entender as formas de acesso, apropriação e uso da informação em ambiente Web.

A população ou universo da pesquisa incluiu as Bibliotecas que possuem as mesmas características definidas para este estudo: Bibliotecas Digitais. A amostra, a parte da população ou do universo selecionada de acordo com o plano de trabalho preestabelecido

A Ciência da Informação através deste estudo tem por objetivo responder está centrada em identificar o modelo de acesso, apropriação e uso da informação utilizada pelos usuários da informação na Web de Dados. Os resultados deste estudo poderão servir de base para a orientação de novos Serviços ao Usuário propostos pela Biblioteca ou Centro de Informação no cenário fortemente dependente da Web, da Ciência de Dados, do Big Data, da Mineração e Análise de Dados, do Cloud Computing, da Inteligência Artificial, da Organização da Informação e do Conhecimento, e da Tecnologia da Informação.

Os desafios tecnológicos impostos pela Era Digital à Ciência da Informação impõem uma mudança urgente de paradigma, uma abordagem tecnológica e preocupação constante com a formação dos profissionais com foco em desenvolver competências alinhadas com as demandas da transformação digital.

\section{CONCLUSÃO}

Como resultado deste estudo espera-se uma contribuição da Ciência da Informação para o entendimento dos recursos da tecnologia colaborativa utilizados em ambientes informacionais digitais. Com base nos recursos identificados e coletados em bibliotecas e repositórios digitais deseja-se observar como são aplicados os recursos da tecnologia colaborativa no contexto da Web Semântica ou Web Inteligente também chamada de Web de Dados. A inserção dessas tecnologias apresenta-se como inovação que devem estar vinculadas à tradição e a missão das bibliotecas e dos repositórios de informação na gestão e organização do conhecimento em sistemas abertos objetivando propor um modelo de Governança da Tecnologia da Informação para a Biblioteca Digital. Avaliar a flexibilidade das estruturas computacionais, sua atratividade e dinâmica na qual o usuário torna-se agente na construção de seu ambiente, demandando recursos de customização e personalização na criação de Serviços de Informação inovadores e que permitam que a Biblioteca continue a ocupar papel relevante na produção de novos conhecimentos por parte de seus Usuários.

Para muitas Bibliotecas Digitais, a crescente disponibilização das tecnologias 
tem demonstrado uma ambigüidade em seu gerenciamento. No aspecto positivo, estas novas tecnologias têm ajudado a aumentar a produtividade dos profissionais da informação, aprimorar o processo de tomada de decisão e acentuar a satisfação do usuário da informação. Porém, a gestão e o suporte destes ambientes heterogêneos e complexos - repletos de diferentes PCs, desktops, dispositivos móveis, impressoras, redes e aplicativos comprovadamente têm se revelado difíceis e dispendiosos para os departamentos de Tecnologia da Informação. Neste contexto torna-se relevante avaliar os principais desafios que as Bibliotecas Digitais terão que enfrentar com relação ao gerenciamento do ciclo de vida de suas tecnologias, consolidação e simplificação de seus processos dentro de seus ambientes computacionais, com objetivo de aumentar a produtividade e construir ambientes ágeis que permitam às Bibliotecas responder as demandas da gestão da informação digital.

Gestores de Bibliotecas Digitais estão cada vez mais envolvidos em maior ou menor grau, no desenvolvimento, controle e monitoração dos ativos tecnológicos de sua organização. A constante pressão por manter os investimentos de $\mathrm{Tl}$ eficiente faz que seja prioritário administrar estes ativos de duas formas: como função do departamento de TI bem como parte de um processo integral da Biblioteca Digital.

A inserção dessas tecnologias apresenta-se como inovação que devem estar vinculadas à tradição e a missão das Bibliotecas e dos repositórios de informação. Avaliar a flexibilidade das estruturas computacionais, sua atratividade e dinâmica na qual o usuário torna-se agente na construção de seu ambiente, demandando recursos de customização e personalização na criação de Serviços de Informação inovadores sejam processos ou serviços, que permitam que a Biblioteca continue a ocupar papel relevante na mediação e produção de novos conhecimentos.

\section{REFERÊNCIAS}

Belling, A. (2010). Exploring Library 3.0 and beyond. 2010. Disponível em: $<$ http://www.libraries.vic.gov.au/downloads/20102011 Shared Leadership Pr ogram Presentation Day /exploring library 3.pdf> Acesso em: 17 maio 2018.

Bruce, C. Seven Faces of Information Literacy. 2003. Disponível em: $<$ http://www.christinebruce.com.au/informed-learning/seven-faces-ofinformation-literacy-in-higher-education/>Acesso em: 16 jun. 2018.

Canclini, N. G. (2009). Diferentes, desiguais e desconectados. Rio de Janeiro: Editora UFRJ. 
Castells, M. \& Cardoso, G. (2005). The Network Society: From Knowledge to Policy. Washington, DC: Johns Hopkins Center for Transatlantic Relations.

Cater-Steel, A. (2009). Information technology governance and service management frameworks and adaptations. New York: Information Science Reference.

Wui-Gee Tan, Aileen Cater-Steel \& Mark Toleman. (2009) Implementing it Service Management: A Case Study Focussing on Critical Success Factors, Journal of Computer Information Systems, 50:2, 1-12. DOI: $10.1080 / 08874417.2009 .11645379$

Choo, C. W. (2003). A organização do conhecimento: como as organizações usam a informação para criar significado, construir conhecimento e tomar decisões. São Paulo: Editora SENAC.

Comitê Gestor da Internet no Brasil - CGI.br. (2010). Dimensões e características da WEB brasileira: um estudo do .gov.br. 2010. Disponível em: $<$ https://www.ufmg.br/proex/cpinfo/educacao/docs/01b.pdf >. Acesso em: 18 jun. 2018

Freire, I. (2010). Reflexões sobre uma ética da informação na sociedade em rede. Ponto De Acesso, v.4, n.3, p.113-133, 2010.Disponível em: shttp://www.portalseer.ufba.br/index.php/revistaici/article/viewArticle/4518>. Acesso em: 22 jun.2015.

FUJITA, M., S. L. (2005). A Biblioteca digital no contexto da gestão de bibliotecas universitárias: análise de aspectos conceituais e evolutivos para a organização da informação. In: ENCONTRO NACIONAL DE CIÊNCIA DA INFORMAÇÃO, 8. Salvador. Anais eletrônicos... Salvador: Editora da Universidade Federal da Bahia - EDUFBA. 2005. Disponível em:

$<$ http://www.cinform-anteriores.ufba.br/vi anais/docs/MariangelaFujita.pdf>. Accesso em: 17 jun.2018

Harbo, K. \& Hansen, T. V. (2012). Getting to Know Library Users' Needs Experimental Ways to User-centred Library Innovation. LIBER Quarterly. v.21, n.3/4, 2012. Disponivel em:

http://liber.library.uu.nl/index.php/lq/article/view/8031/8392 . Acesso em: 02 mai. 2018.

Harbo, K. \& Biegel, R. A. (2010). Field Trip to Library User Land. 2010 Deff Denmark's Electronic Research Library and Changepilot. Disponivel em: http://www.deff.dk/fileadmin/user_upload/dokumenter/DEFF/Publikationer/And re_rapporter/Brugerkaravanen_UK.pdf . Acesso em: 02 maio. 2018

IFLA. Intelligent Library Buildings. 1997. Disponível em:

<http://www.ifla.org/publications/intelligent-library-buildings>. Acesso em: 12 jun. 2018. 
Kuhlthau, C. (2004). Seeking meaning: A process approach to library and information services. 2 ed. Westport, Conn.: Libraries Unlimited.

Le Coadic, Yves-François. (1996). A ciência da informação. Brasília, DF: Briquet de Lemos.

Lancaster, F. W. (2004). Avaliação de Serviços de Bibliotecas. Brasilia: Briquet de Lemos.

Claveiro, G. M. (2005). La Estrategia de Lisboa sobre la sociedad del conocimiento: la nueva economía. Espanha: Serviços de Publicaciones. Disponível em: <https://repositorio.uam.es/handle/10486/663684> Acesso em: 26 jun. 2018.

OECD. (2005). Oslo Manual: Guidelines for Collecting and Interpreting Innovation Data, 3rd Edition, The Measurement of Scientific and Technological Activities, OECD Publishing. DOI: http://dx.doi.org/10.1787/9789264013100$\underline{\text { en }}$

OECD. (2002). Manual de Frascati: Proposta de Práticas Exemplares para Inquéritos sobre Investigação e Desenvolvimento Experimental, F-Iniciativas, Spain. DOI: http://dx.doi.org/10.1787/9789264065611-pt

Observatório para a Cibersociedad. Ambientes informacionais: as bibliotecas digitais e os repositórios institucionais no contexto da web 2.0. 2009. Disponível em:

<https://dialnet.unirioja.es/servlet/articulo?codigo=3348727>. Acesso em: 10 jun. 2018.

Wilson, T. D. (1981). On User Studies and Information Needs. Journal of Documentation, Vol. 37 Iss: 1 , pp.3 - $15 . \quad$ DOI: http://dx.doi.org/10.1108/eb026702

Witten, I. H \& Bainbridge, D. \& Nichols, D.M. (2009). How to build a digital library. 2 ed. San Francisco, CA: Morgan Kaufmann.

ACKNOWLEDGMENT: FAPESP Research Project - Processo 2016/07358-6 